\title{
Thoracic Oncology HERMES syllabus: setting the basis for thoracic oncology training in Europe
}

\author{
Fernando Gamarra', Paolo Boffetta르. Dirk De Ruysscher ${ }^{3}$, Enriqueta Felip ${ }^{4}$, \\ Mina Gaga ${ }^{5}$, Bogdan Grigoriu ${ }^{6}$, Rudolf M. Huber ${ }^{7}$, Sam M. Janes ${ }^{8}$, \\ Charles Hugo Marquette", Gilbert Massard ${ }^{10}$, Julie-Lyn Carrillo Noel ${ }^{11}$, \\ Jean-Paul Sculier ${ }^{12}$ and Anne-Pascale Meert ${ }^{12}$
}

\begin{abstract}
Affiliations: 'Sektion Pneumologie Innenstadt und Thorakale Onkologie, Medizinische Klinik und Poliklinik 5, Ludwig-Maximilians University, Munich, and ${ }^{7}$ Dept of Medicine, Division of Respiratory Medicine and Thoracic Oncology, University of Munich and Thoracic Oncology Centre Munich, Munich, Germany. ${ }^{2}$ The Tisch Cancer Institute and Institute for Translational Epidemiology, Mount Sinai School of Medicine, New York, NY, USA. ${ }^{3}$ Radiation Oncology, University Hospitals Leuven/KU, Leuven, and ${ }^{12}$ Service des Soins Intensifs et Urgences Oncologiques, Oncologie Thoracique, Institut Jules Bordet (ULB), Bruxelles, Belgium. ${ }^{4}$ Thoracic Oncology Unit, Oncology Dept, Vall d'Hebron University Hospital, Vall d'Hebron Institute of Oncology (VHIO), Barcelona, Spain. ${ }^{57}$ th Respiratory Medicine Dept, Athens Chest Hospital Sotiria, Athens, Greece. ${ }^{6}$ Regional Institute of Oncology, University of Medicine and Pharmacy, lasi, Romania. ${ }^{8}$ Lungs for Living Research Centre, UCL Respiratory, University College London, UK. 'Service de Pneumologie, Centre Hospitalier Universitaire de Nice, and ${ }^{10}$ Service de Chirurgie Thoracique, Hôpitaux Universitaires de Strasbourg. Strasbourg. France. ${ }^{11}$ Educational Activities, European Respiratory Society Headquarters, Lausanne, Switzerland.
\end{abstract}

Correspondence: F. Gamarra, Funktionsoberarzt Pneumologie, Funktionsoberarzt Pneumologische Endoskopie, Medizinische Klinik 5 (Pneumologie), Klinikum der LMU-München, Ziemssenstr. 1, 80336, Munich, Germany. E-mail: gamarrađmed.uni-muenchen.de

0 @ERSpublications

The launch of the Thoracic Oncology HERMES syllabus http://ow.ly/mxF6W

This month, the Thoracic Oncology syllabus is published in Breathe. HERMES (Harmonised Education in Respiratory Medicine for European Specialists) is a European respiratory Society (ERS) initiative with the aim of improving the standards of respiratory medicine training and education and to achieve, by harmonisation, the conditions for more career opportunities in Europe [1].

The Thoracic Oncology HERMES Task Force was launched in September 2011. Being the sixth HERMES project we relied on the experience accumulated by previous groups [2-4]. However the development of the Thoracic Oncology syllabus presented new challenges.

Thoracic oncology is a growing medical specialty. In the last decades, the interest in lung cancer and pleural mesothelioma has rapidly increased. Lung cancer is currently the deadliest cancer [5], but there have been considerable advances in the diagnosis and treatment of this disease that has led to an improvement of median survival in advanced-stage lung cancers from 7-8 months in 2000 [6] to 15 months in 2010 [7]. The incidence of mesothelioma is still increasing worldwide [8].

Acknowledging the importance of thoracic malignancy, the ERS established the Thoracic Oncology Assembly in 2009. A year later the Assembly launched the thoracic oncology action plan [9]. The main objective was the improvement in quality of patient care and medical education. Consequently the

Received: June 052013 | Accepted: June 262013

Conflict of interest: J-L.C. Noel is a current employee for the European Respiratory Society. Further disclosures can be found alongside the online version of this article at www.erj.ersjournals.com 
European Initiative for Quality Management in Lung Cancer Care (EIQMLC) and the Thoracic Oncology HERMES Task Forces were founded.

We were aware that thoracic oncology is still not an officially recognised specialty or subspecialty in many European countries. Therefore, the task force started with a survey to assess the current practice of thoracic oncology in Europe. As for all other surveys mentioned below, the questionnaire was sent to an "expert group" consisting of the task force members, a list of national representatives from the EIQMLC, which included 37 European and seven non-European countries and the national representatives of the Forum of European Respiratory Societies (FERS), in which 35 countries are represented. Subsequently, all members of the Thoracic Oncology Assembly of the ERS were invited to participate in the "public group".

$80 \%$ of the participants responded that management of thoracic oncology conditions by a multidisciplinary team (MDT) is mandatory or recommended in their countries. However, only $29 \%$ stated that thoracic oncology was a recognised specialty or subspecialty. Thoracic oncology training differs between countries, with many requiring both rotations in pulmonary and oncology units.

Making a syllabus for thoracic oncology, which is inherently interdisciplinary, was, therefore, a complex process. Taking for example lung cancer, treatment will consist of just surgery or a multimodal combination of surgery, radiotherapy and chemotherapy/targeted therapy depending on the stage and other factors. Modern systemic treatment will depend on results of immunohistochemistry and molecular pathology requiring an intensive communication between clinicians and pathologists. The same applies for mesothelioma, where a multimodal approach is also necessary. Patients need to have access to specialists of the different disciplines in order to get the best possible treatment. Multidisciplinary teamwork seems to improve survival for example of breast cancer patients [10] and multidisciplinary tumour boards have been advocated as an important tool to improve care of lung cancer [11]. However, the latter remains controversial [12].

We foresee thoracic oncology specialists as part of an interdisciplinary team and not working alone, but being experts in all disciplines. Each thoracic oncology specialist will be an expert in a distinct field, for example interventional bronchoscopy, medical oncology, respiratory medicine, thoracic surgery or radiotherapy, but have enough knowledge of the complementary disciplines in order to discuss diagnosis and management strategies with their colleagues. They will have the interdisciplinary perspective and can lead the multidisciplinary conference.

The composition of the task force reflects the interdisciplinary character of Thoracic Oncology. Together with respiratory physicians a medical oncologist (Enriqueta Felip), a thoracic surgeon (Gilbert Massard), and a radiotherapist (Paul de Ruysscher) were invited to participate. They are active members of the European Society of Medical Oncology, the European Society of Thoracic Surgery and the European Society of Radiation Oncology, respectively.

The task force was determined to address the present syllabus and a future thoracic oncology HERMES diploma, not only for respiratory physicians, but also for medical oncologists, thoracic surgeons and radiotherapists that are willing to get an additional qualification in order to work in a leading position in a specialised thoracic oncology centre.

The Thoracic Oncology HERMES Task Force started during the ERS annual congress in September 2011 (Amsterdam, the Netherlands). The methodology used for developing the syllabus was that of other HERMES projects [1-4]. The first draft of the syllabus was discussed at a plenary meeting defining the modules. The items to be included in each module were formulated by working groups and discussed during a second conference.

As with previous HERMES projects, modified Delphi techniques were used, in order to involve the largest possible number of interested participants from as many countries as possible, in the production of the syllabus. A first Delphi survey was sent to the "experts" and "public" groups as defined above. They had to vote on each module and item of the syllabus indicating if this should be "mandatory" or "optional". The respondents also had the opportunity to suggest additional items or changes in the composition of the modules. The results of the survey were discussed during a further task force meeting and a definitive syllabus draft was prepared. There were almost no discrepancies between the answers or suggestions of the survey respondents and those of the task force members.

The next step was to define "levels of competence" for each item of the syllabus. After several discussions and taking into account the efforts of the HERMES leadership to unify the definitions of "levels of competence" in the different HERMES projects, the task force agreed to use the following version. Level 1: Foundation competency, to be used in daily practice by everybody involved in the field. It is a requisite to function in the multidisciplinary team/setting. Level 2: Intermediate competency, to be demonstrated or 
applied with consultation within the multidisciplinary team. Level 3: Specialised competency, which has the highest level of complexity and specialisation.

The aim of grading the syllabus items was to differentiate between topics that need to be mastered by every thoracic oncology specialist (e.g. knowing if a patient should be offered a lobectomy) and those that are very complex (e.g. techniques in performing a lobectomy and treating specific complications) and, therefore, only intended for a certain group.

A second Delphi survey was started, which asked participants to assign levels of competence for each syllabus item. This time the answers were not as clear as in the first survey. There were many discrepancies between the "expert" and "public" groups and the task force members. For some items the level of competence was decided by a narrow majority of votes.

The method of reaching a broader consensus through a Delphi process has the advantage of including the opinion of many participants from different countries and with a variety of backgrounds. Considering the diversity of medical practice in Europe this seems quite important. A disadvantage was that the wide range of respondents may have different levels of experience in the field. With respect to the "public" group of the Thoracic Oncology Assembly members participation was voluntary and we, therefore, assume that those participating were interested in medical education. The participants in the group of "experts" were mostly active members of the Thoracic Oncology Assembly or FERS representatives. The latter being recognised physicians that have access to reliable information in their respective countries.

HERMES projects have defined levels of competence adapted from the model of MiLLER [13]. He proposed a pyramid to describe the principles of complex assessment tools. His pyramid is divided into four levels labelled from the base to the top. 1) Knows (knowledge). 2) Knows how (competence). 3) Shows how (performance). 4) Does (action).

According to Miller [13] new assessment tools should be developed and validated to assess action and performance and not just knowledge.

However, Miller [13], cites the M. Webster Dictionary defining competence as the "quality of being functionally adequate, or of having sufficient knowledge, judgment, skill, or strength for a particular duty" [13]. KHAN and Ramachandran [14] cite the Oxford English Dictionary defining competence as "an ability to do something successfully or efficiently". They point out that competence is the attribute of a person [14]. These authors also refer to the Dreyfus five-stage model of skills acquisition, which differentiates five levels of performance. 1) Novice. 2) Advanced beginner. 3) Competence. 4) Proficiency. 5) Expertise.

Competence here is a point on the spectrum of performance. The first two levels correspond to training and the others, starting with "competence", to deliver effective clinical professional practice. According to this model, if we aim to grade the level of skill acquisition of a trainee we would be better to use the term "level of performance" instead of "level of competence" [14]. Considering the current definitions and since thoracic oncology specialists are supposed to be experts working in a specialised centre, the third level of performance of the Dreyfus model ("competence") is the minimum required from a student of the thoracic oncology diploma.

The syllabus is basically a list of topics that serves as groundwork in building the curriculum and examination. For the creation of the curriculum, which is the next step, the knowledge, skills, attitudes and behaviour associated with each syllabus item will be defined. Additionally we will give recommendations for training and evaluation of skills, specifying the level of performance that the candidates need to achieve. The Dreyfus model of skill acquisition is probably more suitable for this purpose than the pyramid model proposed by MiLLER [13].

A main challenge will be to account for the different backgrounds of the thoracic oncology diploma candidates. A medical oncologist does not need to achieve the same level of performance in interventional bronchoscopy as a respiratory physician and we obviously don't expect a radiotherapist to perform a lobectomy.

In summary we are announcing the publication of the Thoracic Oncology HERMES syllabus (table 1). As far as we are aware, it is the first of its kind and the first effort to harmonise thoracic oncology training in Europe. The syllabus was developed by the Thoracic Oncology HERMES Task Force and validated through two Delphi surveys, involving representatives of almost all European countries and the members of the Thoracic Oncology Assembly of the ERS.

The next step will be to prepare a curriculum detailing the knowledge, skills, attitudes and behaviours, the training methods as well as the level of performance we will require for each item in the future thoracic 


\section{TABLE 1 Thoracic Oncology HERMES syllabus modules}

A General principles of the biology of thoracic cancers

B Aetiology and epidemiology

C Clinical presentations

D Diagnostic procedures

E Imaging

F Diagnostic and interventional bronchoscopic techniques and medical thoracoscopy

G Clinical and pathological staging

H Pathology of intrathoracic tumours

I Prognostic factors/predicitive markers

J Principles of thoracic surgery

K Management of surgical complications

$\mathbf{L}$ Principles of radiation therapy

M Principles of systemic therapy

$\mathbf{N}$ Side-effects of systemic therapy and their management

0 Combined modality treatments

P Management of particular groups of patients

Q Treatment evaluation and follow-up

R Supportive care

S Methodologies for clinical practice and research

T Ethics

$\mathbf{U}$ Cancer related immunology

V Economic considerations in lung cancer treatment

HERMES: Harmonised Education in Respiratory Medicine for European Specialists.

oncology examination. The curriculum will make explicit recommendations for thoracic oncology training in Europe.

\section{Acknowledgements}

The Thoracic Oncology Task Force would like to thank all the ERS HERMES staff members for their support during the whole project.

\section{References}

1 Loddenkemper R, Severin T, Haslam PL. European curriculum recommendations for training in adult respiratory medicine: crossing boundaries with HERMES. Eur Respir J 2008; 32: 538-40.

2 Artigas A, Pelosi P, Dellweg D, et al. Respiratory critical care HERMES syllabus: defining competencies for respiratory doctors. Eur Respir J 2012; 39: 1294-1297.

3 De Backer W, Simonds AK, Horn V, et al. Sleep HERMES: a European training project for respiratory sleep medicine. Eur Respir J 2011; 38: 496-497.

4 Gappa M, Paton J, Baraldi E, et al. Paediatric HERMES: update of the European Training Syllabus for Paediatric Respiratory Medicine. Eur Respir J 2009; 33: 464-465.

5 Siegel R, Naishadham D, Jemal A. Cancer statistics, 2012. CA Cancer J Clin 2012; 62: 10-29.

6 Schiller JH, Harrington D, Belani CP, et al. Comparison of four chemotherapy regimens for advanced non-smallcell lung cancer. N Engl J Med 2002; 346: 92-98.

7 Paz-Ares LDMF, Dediu M, et al. PARAMOUNT: Final overall survival (OS) results of the phase III study of maintenance pemetrexed (pem) plus best supportive care (BSC) versus placebo (plb) plus BSC immediately following induction treatment with pem plus cisplatin (cis) for advanced nonsquamous (NS) non-small cell lung cancer (NSCLC). J Clin Oncol 2012; 30: Suppl. LBA7507.

$8 \quad$ Raja S, Murthy SC, Mason DP. Malignant pleural mesothelioma. Curr Oncol Rep 2011; 13: $259-264$.

9 Sculier JP, Vansteenkiste J, Schonfeld N, et al. Thoracic oncology in Europe: the ERS action plan by the Thoracic Oncology Assembly. Eur Respir J 2010; 36: 1227-1228.

10 Kesson EM, Allardice GM, George WD, et al. Effects of multidisciplinary team working on breast cancer survival: retrospective, comparative, interventional cohort study of 13722 women. BMJ 2012; 344: e2718.

11 Riquet M, Mordant P, Henni M, et al. Should All Cases of Lung Cancer be Presented at Tumor Board Conferences? Thorac Surg Clin 2013; 23: 123-128.

12 Keating NL, Landrum MB, Lamont EB, et al. Tumor boards and the quality of cancer care. J Natl Cancer Inst 2013; 105: 113-121.

13 Miller GE. The assessment of clinical skills/competence/performance. Acad Med 1990; 65: S63-S67.

14 Khan K, Ramachandran S. Conceptual framework for performance assessment: competency, competence and performance in the context of assessments in healthcare - deciphering the terminology. Med Teach 2012; 34: 920-928. 